\title{
Intervenciones breves con adolescentes estudiantes rurales que consumen alcohol en exceso
}

\author{
Brief interventions with adolescent rural \\ students who drink alcohol in excess
}

Recibido: enero 7 de 2010 | Revisado: marzo 1 de 2010 | Aceptado: abril 15 de 2010

\author{
Martha Leticia SAlazAR DE Garza* \\ José FÉliX VALDEZ RuIZ \\ Kalina Isela Martínez Martínez \\ Francisco Javier Pedroza Cabrera \\ Universidad Autónoma de Aguascalientes, México
}

SICI: 1657-9267(201112)10:3<803:IBCAER > 2.3.TX;2-M

Para citar este artículo. Salazar, G. M. L., Valdez, R. J. F., Martínez, M. K. I. \& Pedroza, C. F. J. (2011). Intervenciones breves con adolescentes estudiantes rurales que consumen alcohol en exceso. Universitas Psychologica, 10 (3), 803-815.

* Centro de Ciencias Sociales y Humanidades. Correos electrónicos: mlsalazar@correo.uaa.mx, jfvaldez@correo.uaa.mx, kimartin@correo.uaa. mx, fjpedroz@correo.uaa.mx

\section{RESUMEN}

Debido a la falta de programas dirigidos a prevenir y tratar el consumo excesivo de alcohol en adolescentes de comunidades rurales, se evaluaron dos modalidades de corta intervención: a) programa breve de cinco sesiones y b) sesión de consejo breve. En una primera fase se realizó un estudio exploratorio, obteniendo un cuadro descriptivo del consumo de alcohol en adolescentes de tres comunidades rurales, mientras que en un segundo momento, mediante un diseño de grupos de comparación, se determinaron los efectos de ambas intervenciones sobre el patrón de consumo y otras variables psicosociales en 24 adolescentes rurales del estado de Aguascalientes (México). Se concluyó que ambas modalidades de intervención son efectivas para el trabajo con adolescentes rurales usuarios de alcohol.

Palabras clave autores

Adolescentes, alcohol, intervenciones breves, zonas rurales, prevención.

Palabras clave descriptores

Sustancias psicoactivas, psicología de la salud, estudio exploratorio.

\section{A B S T R A C T}

Due to the lack of programs designed to prevent and treat problems related to alcohol consumption in adolescents in rural communities, two brief intervention modalities were evaluated: a) a brief program with five sessions and, b) a brief advice session. During the first stage, an exploratory study was undertaken, on the basis of which a descriptive chart of alcohol consumption among adolescents in three rural communities was obtained, while in the second stage, comparison groups were designed to determine the effects of both interventions on consumption patterns and other psychosocial variables in a sample of 24 rural adolescents of the state of Aguascalientes, Mexico. The conclusion was that both interventions are effective in working with rural adolescent alcohol users.

Key words authors

Adolescents, alcohol, brief interventions, rural communities, prevention.

Key words plus

Psychoactive substances, health psychology, exploratory research. 
En México algunos estudios han reportado que el consumo excesivo de alcohol entre los adolescentes rurales, se asocia con problemas con los padres y con la Policía: problemas de salud, conducción en estado de ebriedad y participación en riñas o peleas (Medina-Mora, Rascón, García \& Ezbán, 1986; Medina-Mora et al., 1993; Secretaría de Salud de México [SSA] \& Instituto Nacional de Estadística, Geografía e Informática de México [INEGI], 2002). En particular, algunas investigaciones reportan que los más afectados son los hombres (Calderón, Campillo \& Suárez, 1981; Medina-Mora et al., 1986), lo que puede estar relacionado con las normas que promueven y toleran el consumo. Otras variables relacionadas con problemas en lugares públicos son el tipo de bebida que se consume, las celebraciones de la comunidad, la producción clandestina de alcohol, la carencia de servicios públicos, el inicio del consumo a edades tempranas, así como la existencia de opiniones y conductas contradictorias en relación al consumo (Salazar, 2008).

Aunque fue solo hasta el año 2002 que esta población se tuvo en cuenta en la Encuesta $\mathrm{Na}$ cional de Adicciones (SSA \& INEGI, 2002), obteniéndose que el índice de consumo de alcohol en adolescentes de zonas rurales fue del 14.4\% (543; 197 adolescentes), con un patrón de consumo de alcohol poco frecuente (menos de una vez al mes), no obstante $4.79 \%$ reportó un consumo alto de alcohol (5 o más copas por ocasión). Además, un dato relevante fue que $4.1 \%$ de los adolescentes hombres rurales cumplieron con el criterio de dependencia del Manual Diagnóstico y Estadístico de los Trastornos Mentales (DSM-IV, por su sigla en inglés), proporción mayor a la encontrada en los adolescentes urbanos (3.3\%). Esto, a pesar de que se observó un porcentaje menor de consumo de alcohol en los adolescentes rurales (14.4\%) en comparación con los de zonas urbanas (35\%).

En relación con el consumo de sustancias, en México existen algunos programas de prevención universal y selectiva, tales como el Programa de Prevención de Riesgos Psicosociales "Chimalli" (Castro, Llanes, Margain \& Carreño, 1999), el programa "Yo quiero, yo puedo" propuesto por el
Instituto Mexicano de Investigación de la Familia y Población (IMIFAP) y la Secretaría de Educación Pública (SEP) (Consejo Nacional Contra las Adicciones [CONADIC], 2002), el Modelo de Educación para la Vida y el Trabajo del Instituto Nacional para la Educación de los Adultos [INEA] (CONADIC, 2002) y el programa de intervención y apoyo a las familias que se enfrentan a problemas de consumo excesivo de sustancias en sus hogares (Natera \& Tiburcio, 2007), los que se han aplicado entre algunos grupos de la población rural. Con todo, no existe el reporte de intervenciones específicas para los adolescentes rurales que consumen alcohol en exceso y que presentan problemas correlativos, lo que sugiere la necesidad de contar con programas de prevención indicada para esta población. Sin embargo, este tipo de programas ha sido desarrollado y evaluado con adolescentes escolares de zonas urbanas, siendo el Programa de Intervención Breve para Adolescentes (PIBA) que inician el consumo de alcohol y otras drogas (Martínez, Salazar, Pedroza, Ruiz \& Ayala, 2008) y el Consejo Breve de una sola sesión (Martínez, Pedroza \& Salazar, 2008; Martínez, Pedroza, Vacío, Jiménez \& Salazar, 2008), dos tipos de aproximaciones que han mostrado resultados efectivos, para detectar y atender de manera temprana a los adolescentes que consumen alcohol en exceso. Ambas aproximaciones son intervenciones breves derivadas de un enfoque conductual, que se caracterizan por tener un tiempo breve de duración, incluyendo una evaluación inicial, un asesoramiento breve y el seguimiento de los casos (Rodríguez-Martos, 2002). El PIBA está conformado por cinco sesiones, en las que se establece una meta de consumo (abstinencia o moderación), se analizan situaciones de riesgo, se desarrollan planes de acción para enfrentar dichas situaciones y se revisa el impacto que el consumo de alcohol tiene sobre las metas de vida de los adolescentes. Mientras que el Consejo Breve consiste en una sesión de trabajo de 90 minutos, en la que se retroalimenta al adolescente sobre su patrón de consumo, factores de riesgo y protección personales, se establece una meta de consumo y se desarrollan alternativas de apoyo para el logro de la meta. 
En su aplicación con adolescentes estudiantes urbanos de México, los resultados muestran que estos reducen la cantidad y frecuencia de su consumo, los problemas asociados y sus situaciones de riesgo, e incrementan su autoeficacia situacional (Martínez, Pedroza et al., 2008; Martínez, Pedroza, Vacio et al., 2008; Martínez, Salazar et al., 2008). Estos resultados coinciden con estudios realizados con adolescentes de otros países, en los que se observa que estas modalidades de intervención tienen un efecto en el número de días de consumo, el promedio del consumo y el número de consecuencias relacionadas, alcanzando efectos moderados sobre las comparaciones del patrón de consumo antes y después de las intervenciones, y en comparación con grupos control o alguna otra intervención (Winters, Leitten, Wagner \& O'Leary, 2007).

Sin embargo, a fin de incrementar la validez externa de ambas intervenciones, es necesario evaluarlas con diferentes poblaciones, tal como la de adolescentes rurales. Además, la adaptación de estas intervenciones resulta conveniente, debido al bajo costo y al menor tiempo que se invierte en su aplicación, ya que en la mayoría de las ocasiones las comunidades rurales no cuentan con los recursos económicos y humanos indispensables para la aplicación de intervenciones más largas.

Por lo antes expuesto, en este trabajo se propuso evaluar el PIBA y el Consejo Breve de una sesión en una muestra de adolescentes pertenecientes a zonas rurales del estado de Aguascalientes en México, a fin de determinar sus efectos sobre la cantidad de consumo, el nivel de riesgo, los problemas relacionados y la autoeficacia para enfrentar diferentes situaciones de consumo. Teniendo como condiciones importantes del estudio, la exploración previa de las características del consumo entre los adolescentes rurales y la adaptación de ambas intervenciones a las características de la población. El estudio comprendió dos fases: 1) exploración de las características del consumo en los adolescentes rurales y 2) evaluación de las intervenciones. A continuación, se describe el método, los resultados y la discusión para cada fase del trabajo.

\section{Fase 1: Exploración de las características del consumo en los adolescentes rurales}

\section{Método}

Esta fase tuvo el objetivo la obtención un cuadro descriptivo del consumo de alcohol entre los adolescentes de tres comunidades rurales, mediante la percepción de informantes de la comunidad.

\section{Participantes}

Se entrevistaron 18 grupos de informantes con un total de 91 sujetos. En la Tabla 1 se muestra el número de participantes de acuerdo a la manera en cómo fueron integrados los grupos en cada comunidad.

\section{TABLA 1}

Datos sociodemográficos de los informantes

\begin{tabular}{|c|c|c|c|c|}
\hline \multicolumn{2}{|r|}{ Variables } & \multirow{3}{*}{$\begin{array}{c}\begin{array}{c}\text { Adoles- } \\
\text { centes } \\
\text { estudiantes }\end{array} \\
48.1 \% \\
51.9 \%\end{array}$} & \multirow{3}{*}{$\begin{array}{c}\begin{array}{c}\text { Adoles- } \\
\text { centes no } \\
\text { estudiantes }\end{array} \\
41.7 \% \\
58.3 \%\end{array}$} & \multirow{3}{*}{$\begin{array}{c}\begin{array}{c}\text { Adul- } \\
\text { tos }\end{array} \\
57.1 \% \\
42.9 \%\end{array}$} \\
\hline$\stackrel{x}{x}$ & Mujer & & & \\
\hline œ & Hombre & & & \\
\hline 苞 & Promedio & 13.9 años & 16.2 años & $\begin{array}{l}39.6 \\
\text { años }\end{array}$ \\
\hline \multirow{4}{*}{$\begin{array}{l}\vec{\Sigma} \\
\dot{U} \\
\dot{0} \\
\vec{I}\end{array}$} & Soltero & $100 \%$ & $100 \%$ & $3.6 \%$ \\
\hline & Casado & & & $89.3 \%$ \\
\hline & Divorciado & & & $3.6 \%$ \\
\hline & Unión Libre & & & $3.6 \%$ \\
\hline \multirow{2}{*}{ 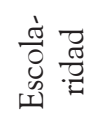 } & Primaria & & $16.6 \%$ & $82.1 \%$ \\
\hline & Secundaria & $100 \%$ & $83.4 \%$ & $17.9 \%$ \\
\hline \multirow{8}{*}{ 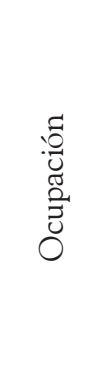 } & Estudiante & $74.1 \%$ & & \\
\hline & Estudiante y & $25.9 \%$ & & \\
\hline & empleado & & & \\
\hline & hogar & & $44.5 \%$ & $53.6 \%$ \\
\hline & Empleado & & & $10.7 \%$ \\
\hline & Oficio & & $27.8 \%$ & $32.1 \%$ \\
\hline & Ninguna & & $22.2 \%$ & \\
\hline & Jubilado & & $5.6 \%$ & $3.67 \%$ \\
\hline
\end{tabular}

Fuente: elaboración propia. 


\section{Definición de Variables}

- Características sociodemográficas de los adolescentes consumidores: sexo, edad y ocupación.

- Razones del consumo: motivos a los que se atribuye el consumo.

- Condiciones que acompañan el consumo: lugares, situaciones de consumo y personas con las que se consume.

- Tipos de sustancias consumidas: tipo de bebida alcohólica consumida.

- Normas relacionadas: reglas a las que se ajusta el consumo de alcohol.

- Percepción de severidad del consumo: opinión de la comunidad acerca la embriaguez entre los adolescentes.

- Pronóstico de los adolescentes que abusan del alcohol: opinión sobre lo que puede ocurrir en el futuro con los adolescentes que consumen alcohol en exceso.

- Alternativas de acción existentes y de acción propuestas: medidas que se toman en la comunidad con los adolescentes que toman en exceso y opinión sobre las acciones que se podrían instrumentar.

\section{Instrumentos}

\section{Historietas}

Con el propósito de que los participantes diferenciaran entre un adolescente consumidor de alcohol en exceso y una persona con adicción, se utilizaron dos historietas que describían ambas situaciones, que fueron validadas a través de juicios dicotómicos alcanzando un $85 \%$ de acuerdo entre los jueces.

\section{Cuestionario}

Con el objetivo de evaluar las variables descritas anteriormente, se empleó un cuestionario compuesto por 33 preguntas con opciones de respuesta múltiple. Este cuestionario fue realizado con base en la entrevista propuesta por Jellinek's (Popham,
1976). La validez se obtuvo mediante juicios dicotómicos. El grado de acuerdo entre los jueces para cada reactivo fue de por lo menos el $85 \%$.

\section{Diseño}

En esta fase del estudio se realizó un estudio de tipo exploratorio mediante la Técnica del Informante (Popham, 1976), la cual consiste en obtener datos a partir de personas que se reúnen en sesiones de dos horas, para discutir y llegar a un consenso sobre las variables evaluadas.

\section{Procedimiento}

En un primer momento, con base en las sugerencias de las autoridades de seguridad pública de Aguascalientes, se seleccionaron tres comunidades rurales en tres diferentes municipios. Enseguida, se capacitó a los coordinadores de los grupos en el manejo de los instrumentos y la conducción de los grupos, quienes eran egresados de la Licenciatura en Psicología. Posteriormente, se integraron seis grupos de informantes por cada comunidad, integrados con base en el sexo y edad de las personas. Para la integración de los grupos de informantes de personas adultas y de adolescentes no estudiantes, se acudió con los líderes de las comunidades, mientras que para los grupos de adolescentes estudiantes, se acudió a las tele-secundarias y telebachilleratos de las distintas comunidades. Los días y horarios de reunión de los grupos se programaron de acuerdo a los tiempos disponibles de los informantes.

\section{Análisis de resultados}

El análisis de resultados se realizó considerando el total de los grupos entrevistados, sin diferenciar entre las características sociodemográficas de los diferentes grupos conformados, ni por la comunidad a la que pertenecían. Para esto se determinaron categorías de las respuestas dadas para cada pregunta y se obtuvo el porcentaje de grupos por cada respuesta. 


\section{Resultados}

\section{Características sociodemográficas} de los adolescentes consumidores

El $88.8 \%$ de los grupos reportó que los hombres se involucran más en el consumo y $11.1 \%$ acordó que hombres y mujeres beben alcohol de igual manera.

En cuanto a la edad, $83.3 \%$ de los grupos expresó que los adolescentes consumidores de alcohol tienen entre 15 y 19 años de edad; $5.5 \%$ acordó que el consumo se da a partir de los 11 años, 5.5 $\%$ desde los 14 años y $5.5 \%$ que inician después de los 20 años.

En cuanto a la ocupación, 50 \% de los grupos acordó que quienes toman más son los que solo trabajan, $44.4 \%$ que los que no hacen nada y $5.5 \%$ expresó que todos los jóvenes, sin importar su ocupación, toman en exceso.

\section{Razones del consumo}

El 94.4\% de los grupos reportó que los adolescentes consumen alcohol en exceso debido a la presencia de problemas familiares, $44.4 \%$ expresó que el consumo es visto como una forma de diversión y $38.8 \%$ argumentó que es una forma de convivir con los amigos.

\section{Principales tipos de sustancias consumidas}

El 94.4\% de los grupos reportó que la cerveza es el principal tipo de bebida de consumo, $77.7 \%$ dijo que los destilados y $16.6 \%$ informó el consumo de alcohol de $96^{\circ}$.

\section{Condiciones que acompañan el consumo}

Para el $66.6 \%$ de los grupos la calle fue el principal lugar de consumo, el $50 \%$ acordó que son las fiestas privadas y $44.4 \%$ reportó que los bailes. Además, $72.2 \%$ expresó que los adolescentes consumen alcohol en compañía de sus amigos, $11.1 \%$ dijo que lo hacen acompañados de sus familiares y $5.5 \%$ expresó que beben acompañados por los dueños de los expendios de bebidas alcohólicas.
Por último, $55.5 \%$ de los grupos mencionó que una de las principales situaciones de consumo son las emociones desagradables, 50 \% expresó que las emociones agradables y $27.7 \%$ enunció a los momentos agradables con otros.

\section{Patrones de consumo}

En relación con los adolescentes hombres, $72.2 \%$ de los grupos reportó que estos beben más de cinco tragos por ocasión de consumo, $16.6 \%$ acordó que ingieren de tres a cinco tragos y $5.5 \%$ dijo que toman menos de tres tragos por ocasión. La mayoría de los grupos $(72.2 \%)$ acordó que los hombres se embriagan dos veces por semana (fines de semana), $22.2 \%$ expresaron que lo hacen en ocasiones especiales y $5.5 \%$ que beben cada quince días.

En cuanto a las mujeres, $38.8 \%$ de los grupos de informantes reportó que estas beben de tres a cinco tragos, $33.3 \%$ expresó que toman menos de tres tragos, $5.5 \%$ dijo que las mujeres toman más de cinco tragos y $22.2 \%$ dijo que no saben en qué cantidad beben las mujeres. Sobre la frecuencia con que las adolescentes se embriagan, $55.5 \%$ de los grupos señaló que lo hacen en ocasiones especiales, $16.6 \%$ acordó que lo hacen los fines de semana, $11.1 \%$ reportó que una vez al año, $5.5 \%$ manifestó que las mujeres nunca se embriagan y $11.1 \%$ no saben la frecuencia con la que las adolescentes se emborrachan.

\section{Normas que favorecen el consumo entre los adolescentes}

El $72.2 \%$ de los grupos acordó que los jóvenes de la comunidad consideran que existen diferencias entre mujeres y hombres, por las que las mujeres no deben tomar, mientras que 27.7 \% manifestó que los jóvenes de la comunidad no consideran que existan diferencias entre hombres y mujeres. También 44.4 \% reportó que los jóvenes de las comunidades no ven a la capacidad de beber en exceso como un asunto de admiración y respeto, pero $27.7 \%$ dijo que los adolescentes sí lo ven de esta forma y otro $27.7 \%$ declaró que es visto de esta manera por los hombres, pero no por las 
mujeres. Por último, 55.5 \% de los grupos reportó que rechazar un trago de alcohol es visto principalmente como 'falta de hombría' o una ofensa, aunque $16.6 \%$ exteriorizó que esto es visto con respeto. Además, $61.1 \%$ de los grupos dijo que los adolescentes ven a los jóvenes que no beben con admiración o aprobación, pero 27.7 \% opinó que, en algunas ocasiones, también son vistos con rechazo.

\section{Percepción de la severidad del consumo}

El 50 \% reportó que la embriaguez entre los adolescentes es vista por la comunidad como un problema grave, $22.2 \%$ que es grave sobre todo en las mujeres, otro $22.2 \%$ acordó que es visto como un problema de poca importancia y 5.5 \% no llegó al consenso.

\section{Pronóstico de los adolescentes que abusan del alcohol}

El 94.4\% convino que los adolescentes que consumen alcohol en exceso pueden empeorar en el futuro y $5.5 \%$ no llegó al consenso. De igual manera, $94.4 \%$ concertó que los adolescentes requieren ayuda para cambiar y $5.5 \%$ dijo que estos jóvenes no necesitan ayuda. Además, el $100 \%$ de los grupos declaró que los jóvenes pueden cambiar su consumo solo si tienen la disposición para hacerlo.

\section{Alternativas de atención existentes $y$ de acción propuestas}

El $100 \%$ de los grupos coincidió en que las principales acciones que se realizan para atender a los adolescentes son: permanecer sin hacer algo al respecto, orientación o consejo por parte de la familia, vigilancia por parte de sus padres, acudir a centros de rehabilitación y llamar a la Policía. Mientras que las sugerencias que los informantes dieron para atender a los jóvenes fueron: rehabilitarlos, llevarlos a grupos como AA o a profesionales, ayudarlos (familiares o amigos), orientarlos o darles algún consejo.

\section{Discusión}

La inclusión de una fase exploratoria en el estudio se debió a que la adaptación de intervenciones desarrolladas y evaluadas para habitantes de zonas urbanas a habitantes de zonas rurales, requiere determinar tanto las características de los individuos que se involucran con el consumo de las diferentes sustancias, como las principales drogas de consumo y las particularidades de las comunidades (Leukefeld, Godlaski, Hays \& Clark, 1999).

De acuerdo al reporte de los grupos de informantes, se encontró que, en las comunidades seleccionadas, los hombres participaban más en el consumo, que los estudiantes constituían uno de los grupos que se encontraban en riesgo para el consumo excesivo de alcohol, aunque los grupos de mayor riesgo eran los que solo trabajaban o los que no hacían nada. Asimismo, se identificó que el grupo de edad en que se presentaba más el consumo era el de 15 a 19 años y que los amigos son las personas con las que se consume con mayor frecuencia. Datos que coinciden con algunos estudios realizados con adolescentes en los que se reporta que el ser hombre, no estudiar de tiempo completo o no trabajar el año previo a la entrevista, estar en el bachillerato (rango de edad de 15 a 19 años) y tener amigos que consumen alcohol y otras drogas, son factores de riesgo para el consumo de sustancias (Medina-Mora et al., 1986; MedinaMora et al., 1993; SSA \& INEGI, 2002).

Por otra parte, se identificó que tanto las razones y situaciones de consumo estaban relacionadas con la presencia de estados emocionales placenteros y desagradables, tal como fue reportado en un estudio previo, cuyo objetivo fue identificar algunas situaciones asociadas con el consumo de alcohol entre los adolescentes rurales (Salazar, Álvarez, Galarza \& Martínez, 2008).

También se identificó que la cerveza y los destilados son dos de los tipos de bebida de mayor consumo, tal como se observa a nivel nacional (SSA \& INEGI, 2002). Pero, de la misma manera, se obtuvo el reporte de consumo de alcohol de $96^{\circ}$. Lo que pudiera deberse al bajo costo de este tipo 
de bebida, coincidiendo con el reporte de algunos estudios en los que se observa una relación entre el tipo de sustancia de consumo y el nivel socioeconómico de las personas (Medina-Mora et al., 1995).

Igualmente, se encontró que tanto los hombres como las mujeres consumían alcohol en cantidades excesivas, incluso mayores a las reportadas en las encuestas nacionales para el grupo de adolescentes rurales. Aunado a esto, se observó que al igual que en otros estudios (Calderón et al., 1981; MedinaMora et al., 1986), el consumo excesivo de alcohol entre los hombres se promueve y se tolera más, así como la presencia de conductas y opiniones contradictorias en relación al consumo excesivo de alcohol y al rechazo del consumo.

Por último, se identificó la falta de servicios psicológicos para la atención de estas comunidades, coincidiendo con el reporte de algunos trabajos en los que se señala que los principales recursos de apoyo en estos lugares, son la familia, los amigos, sacerdotes, entre otros (Berenzon, Medina-Mora \& Lara, 2003). Al mismo tiempo, se observó que las comunidades reconocían que los profesionales o los centros de rehabilitación pueden participar en la atención de estos jóvenes.

Una vez concluida esta fase, se determinó que la evaluación de las intervenciones se realizaría con adolescentes estudiantes, ya que este era el grupo para el que originalmente se desarrollaron las intervenciones, y debido a que la falta de estructura física en materia de salud, impedía la difusión y aplicación entre adolescentes no estudiantes.

\section{Fase 2: Evaluación de las intervenciones}

\section{Método}

El objetivo de esta fase fue aplicar dos modalidades de intervención breve, a fin de determinar sus efectos sobre la cantidad de consumo, nivel de riesgo, problemas asociados y autoeficacia situacional.

\section{Participantes}

Fue una muestra no probabilística de 23 sujetos voluntarios usuarios de alcohol, asignados aleato- riamente, 12 al PIBA y 11 al Consejo Breve. Los criterios de inclusión fueron: a) tener un nivel de riesgo de consumo de alcohol moderado, alto o muy alto en la línea base, b) reportar problemas relacionados con el consumo, c) tener entre 14 y 18 años de edad y d) ser estudiante regular e inscrito. En la Tabla 2 se observan las principales características sociodemográficas de los participantes por condición de tratamiento.

\section{TABLA 2}

Datos sociodemográficos de los participantes en cada modalidad de intervención

\begin{tabular}{|c|c|c|}
\hline & $\begin{array}{c}\text { Programa } \\
\text { de cinco } \\
\text { sesiones } \\
\text { [PIBA] }\end{array}$ & $\begin{array}{c}\text { Consejo } \\
\text { Breve }\end{array}$ \\
\hline Sexo & $\begin{array}{l}\text { Hombres } \\
50 \% \\
\text { Mujeres } \\
50 \%\end{array}$ & $\begin{array}{l}\text { Hombres } \\
36.4 \% \\
\text { Mujeres } \\
63.6 \%\end{array}$ \\
\hline Edad promedio & 15.4 años & 15.9 años \\
\hline Nivel de escolaridad & $\begin{array}{l}\text { Secundaria } \\
50 \% \\
\text { Bachillerato } \\
50 \%\end{array}$ & $\begin{array}{l}\text { Secundaria } \\
27.3 \% \\
\text { Bachillerato } \\
72.7 \%\end{array}$ \\
\hline Ocupación & $\begin{array}{l}\text { Estudiante } \\
66.7 \% \\
\text { Estudiante } \\
\text { y trabajador } \\
33.3 \%\end{array}$ & $\begin{array}{l}\text { Estudiante } \\
90.9 \% \\
\text { Estudiante } \\
\text { y trabajador } \\
9.1 \%\end{array}$ \\
\hline
\end{tabular}

Fuente: elaboración propia.

\section{Definición de Variables}

- Patrón de consumo: número de tragos estándar consumidos.

- Nivel de riesgo: riesgo en que se encuentra un sujeto para la ocurrencia de problemas agudos ocasionados por su consumo de alcohol.

- Problemas relacionados: consecuencias negativas asociadas con el consumo de alcohol.

- Autoeficacia situacional: percepción que tiene un individuo sobre su capacidad para controlar su consumo. 


\section{Instrumentos}

Cuestionario de Tamizaje de los

Problemas en Adolescentes [POSIT]

(Mariño, González-Forteza, Andrade

\& Medina-Mora, 1997)

Cuestionario integrado por 81 reactivos que contemplan siete áreas de funcionamiento, aunque para el propósito de este estudio solo se utilizó el área de abuso de sustancias que evalúa consecuencias negativas asociadas con el consumo de alcohol y otras drogas, en una escala de respuesta dicotómica. En la adaptación del cuestionario a población de adolescentes mexicanos, se determinó un alfa de Cronbach de 0.91.

Linea Base Retrospectiva

[LIBARE] (Sobell, Maisto,

Sobell \& Cooper, 1979)

Es un registro de los tres meses previos a la evaluación, con el objetivo de identificar el patrón de consumo en cuanto a frecuencia y cantidad de consumo en un día típico. La confiabilidad y validez de este instrumento han sido establecidas en poblaciones de adultos y adolescentes usuarios de alcohol y de drogas ilegales, mediante la comparación con marcadores biológicos y el reporte de colaterales.

Autorregistro de Consumo de Alcohol y otras Drogas (Ayala, Cárdenas, Echeverría \& Gutiérrez, 1998)

El autorregistro es un formato donde el sujeto reporta el día de consumo, la capacidad de resistir el consumo, el número de copas por tipo de bebida, la situación relacionada con el consumo, los pensamientos y sentimientos relacionados con el consumo. La validez del autorregistro se obtiene de su aplicación clínica al encontrar un patrón similar, cuando el autorregistro es comparado con reportes de otros informantes y con reportes escolares.
Cuestionario de Confianza Situacionalversión breve [CCS] (Ayala et al., 1998)

Su objetivo es medir la autoeficacia de los individuos en relación con su percepción para controlar el consumo en diferentes situaciones de riesgo, en ocho diferentes situaciones de riesgo para el consumo, en una escala de respuesta de 0 a $100 \%$. En su validación con adolescentes mexicanos, obtuvo una consistencia interna de 0.97 , medida a partir del alfa de Cronbach.

\section{Diseño}

Se utilizó un diseño de grupos de comparación (Kazdin, 2001), en el que se compararon dos tratamientos que se consideran apropiados para la atención de adolescentes que se inician en el consumo de alcohol y presentan problemas relacionados, sin que ninguno de ellos represente un tratamiento estándar para el trabajo con adolescentes rurales.

\section{Procedimiento}

El primer paso consistió en la adaptación de los instrumentos y materiales. Enseguida, se pilotearon ambas intervenciones, para lo que se contó con la participación de seis adolescentes rurales usuarios de sustancias, tres de ellos recibieron la sesión de Consejo Breve y tres, el PIBA. Tanto los adolescentes que participaron en el piloteo como los que participaron en la aplicación final, habitaban en las comunidades seleccionadas durante la fase previa. La muestra final se detectó mediante la instrumentación de pláticas preventivas en las que al final se aplicó el POSIT. Los adolescentes que reportaron al menos una consecuencia relacionada con el consumo de alcohol en este cuestionario, fueron entrevistados de manera individual para determinar si cumplían con los criterios de inclusión, momento en que se utilizó la LIBARE y el CCS. Posteriormente, los adolescentes fueron asignados de manera aleatoria para recibir cualquiera de las modalidades de intervención. En la Tabla 3 se presenta la organización de cada una de las modalidades de intervención. 
TABLA 3

Estructura de ambas modalidades de intervención

\begin{tabular}{ll}
\hline $\begin{array}{c}\text { Programa Breve de cinco } \\
\text { sesiones [PIBA] }\end{array}$ & $\begin{array}{c}\text { Consejo Breve de una } \\
\text { sesión }\end{array}$ \\
\cline { 1 - 2 } Dos sesiones de evaluación & \multicolumn{1}{c}{$\begin{array}{c}\text { Dos sesiones de } \\
\text { evaluación }\end{array}$} \\
\cline { 1 - 2 } tratamiento &
\end{tabular}

Fuente: elaboración propia.

Durante la fase de tratamiento y seguimiento se utilizó el autorregistro, a fin de mediar la cantidad de consumo en ambas fases del estudio. La evaluación final se tuvo un mes después de concluida la intervención y los seguimientos se realizaron a los tres y seis meses después del tratamiento.

\section{Análisis de resultados}

Se realizaron análisis con la prueba $U$ de MannWhitney para muestras independientes, en cuanto al número de tragos ingeridos y el número de problemas reportados durante la evaluación inicial y en el seguimiento. Se emplearon pruebas de Friedman para comparar el promedio de tragos ingeridos durante las diferentes fases de la intervención de acuerdo a la modalidad de intervención, y la prueba de Kruskal-Wallis para confrontar ambos grupos de intervención en los tres períodos de registro. También se calculó el tamaño del efecto para cada condición con base en la fórmula propuesta por Cohen (Clark-Carter, 2002). Además, se utilizó la prueba de rangos de Wilcoxon para comparar el nivel de riesgo, el número de problemas relacionados y la autoeficacia situacional entre las fases de evaluación y seguimiento.

\section{Resultados}

\section{Diferencias entre los grupos en la fase de línea base}

El análisis realizado con la prueba $U$ de MannWhitney para muestras independientes no mostró diferencias significativas entre la cantidad de tragos bebidos durante la línea base entre los grupos $U$ ( $n$ $=23)=0.092, p>0.05$. El promedio de tragos consumidos en la evaluación se obtuvo con la LIBARE. La mayoría de los adolescentes del PIBA bebió en promedio 41.20 tragos, mientras que los de Consejo Breve consumieron 48.90 tragos. Tampoco se encontraron diferencias estadísticas significativas entre los grupos en relación con el número de problemas reportados durante la evaluación $U(n=23)=0.062, p>0.05$. Los adolescentes asignados al PIBA reportaron en promedio 6.16 problemas y los de Consejo Breve, 8.45. El número de problemas relacionados se obtuvo mediante el POSIT.

\section{Efecto de las intervenciones sobre el patrón de consumo y nivel de riesgo}

Los adolescentes asignados al PIBA durante la línea base tuvieron un promedio de 41.20 tragos estándar $(D E=20.16)$, de 9.08 tragos estándar $(D E=$ 12.88) en el primer seguimiento y de 6.75 tragos estándar $(D E=11.74)$ en el segundo seguimiento. Por su parte, los asignados al Consejo Breve en la línea base alcanzaron un promedio de 48.90 tragos estándar, de 16.00 tragos estándar $(D E=15.63)$ 
en el primer seguimiento y de 16.90 tragos estándar $(D E=15.04)$ en el segundo seguimiento. El promedio de tragos consumidos durante los seguimientos se obtuvo mediante el autorregistro del consumo.

Los resultados de las prueba de Friedman mostraron que los jóvenes de ambas intervenciones, disminuyeron la cantidad promedio de consumo de la fase de línea base a la de seguimiento; el grupo del PIBA obtuvo $c^{2} \mathrm{~F}(n=12)=12.977, p<0.01$, y el de Consejo Breve $c^{2} \mathrm{~F}(n=11)=14.000, p<$ 0.01 (véase Figura 1).

Por otra parte, el análisis de Kruskal-Wallis demostró que no hubo diferencias estadísticas significativas entre ambos grupos de intervención en ninguno de los tres períodos registrados: línea base $H(n=11)=0.009, p>0.05$, primer seguimiento $H(n=11)=1.062, p>0.05$, y segundo seguimiento $H(n=11)=3.392, p>0.05$.

Para el cálculo del tamaño del efecto se obtuvo una desviación estándar combinada, la cual fue de 25.38. De esta manera, el tamaño del efecto encontrado para el grupo que recibió el PIBA fue $d=0.69$ y para el grupo de Consejo Breve fue $d=$ 0.69 , lo que significó que ambos grupos tuvieron un tamaño del efecto mediano.
Por último, se analizaron las diferencias en el nivel de riesgo para la ocurrencia de problemas agudos con base en los criterios de la Organización Panamericana de la Salud/Oficina Regional de la Organización Mundial de la Salud [OMS] (2000). Los niveles de riesgo en un día de consumo para los hombres son: bajo riesgo, de una a cuatro copas; riesgo moderado, entre cinco y seis copas; alto riesgo, de siete a diez copas y muy alto riesgo, más de diez copas. Mientras que para las mujeres son: bajo riesgo, de una a dos copas; riesgo moderado, de tres a cuatro copas; riesgo alto, de cinco a seis copas y muy alto riesgo, más de seis copas. Estos datos se obtuvieron de la LIBARE y el autorregistro, dividiendo la cantidad total de tragos ingeridos entre el número de días de consumo. El análisis realizado con la prueba de rangos de Wilcoxon, mostró diferencias significativas para ambos grupos de la línea base al segundo período de seguimiento. Tanto el grupo que recibió el PIBA como el grupo de Consejo Breve en la línea base, alcanzaron un nivel de riesgo alto, mientras que en el segundo período de seguimiento obtuvieron un nivel de riesgo bajo $T$ $(n=12)=2.625, p<0.05$ y $\mathrm{T}(n=11)=2.754$, $p<0.05$, respectivamente.

Figura 1

Tragos estándar consumidos en las diferentes fases del estudio

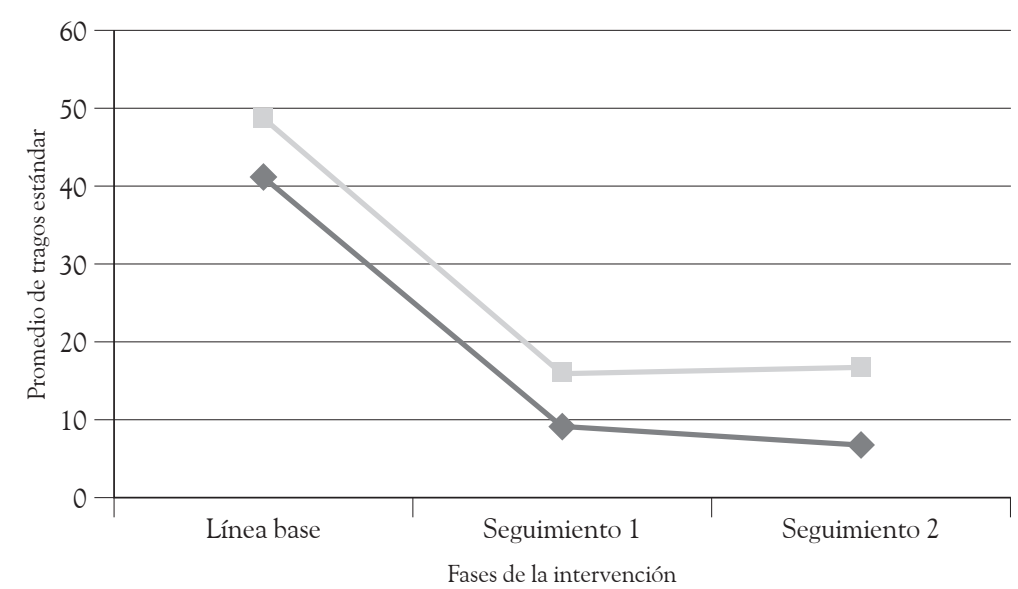

Programa de cinco sesiones - Consejo breve

Fuente: elaboración propia. 


\section{Efecto de las intervenciones sobre el} número de problemas relacionados

El análisis realizado mediante la prueba de rangos de Wilcoxon, arrojó diferencias estadísticas significativas entre el número de problemas reportados antes y después del tratamiento, tanto para el grupo que recibió el PIBA T $(n=12)=3.064, p<0.05$, como el de Consejo Breve $T(n=11)=1.962, p$ $=0.05$. Por su parte, la comparación realizada con la prueba $U$ de Mann-Whitney, no mostró diferencias estadísticas significativas entre el número de problemas reportados durante el seguimiento por ambos grupos $U(n=23)=-2.034, p>0.05$. El grupo que recibió el PIBA tuvo 6.16 problemas en promedio en evaluación y 0.45 durante el seguimiento, mientras que el grupo de Consejo Breve reportó en promedio 8.45 problemas en evaluación y 2.72 en el seguimiento. El número de problemas relacionado se obtuvo mediante la aplicación del POSIT.

\section{Efecto de las intervenciones sobre el nivel de autoeficacia situacional}

En cuanto al nivel de autoeficacia situacional, se comparó el porcentaje de confianza reportado por los adolescentes en las ocho escalas del CCS durante la fase de evaluación y el segundo período de seguimiento. Para el análisis, se consideraron cuatro niveles de riesgo situacional: 1) riesgo bajo, 2) riesgo moderado, 3) riesgo alto y 4) riesgo muy alto. La prueba de rangos de Wilcoxon, mostró diferencias significativas para el grupo que recibió el PIBA en las situaciones de: emociones desagradables $T(n=12)=2.414, p<0.05$, probando control $T(n=12)=2.041, p<0.05$, necesidad física $T(n=12)=2.271, p<0.05$, conflictos con otros $T(n=12)=2.070, p<0.05$ y momentos agradables con otros $T(n=12)=2.126, p<0.05$. Mientras que el grupo que recibió el Consejo Breve, alcanzó cambios significativos en las escalas de: emociones desagradables $T(n=11)=2.232, p<$ 0.05 , emociones agradables $T(n=12)=2.041, p$ $<0.05$, necesidad física $T(n=12)=1.983, p<$
0.05, presión social T $(n=12)=2.280, p<0.05$ y momentos agradables con otros $T(n=12)=$ 2.850, $p<0.05$.

\section{Discusión}

En la segunda fase, antes de la aplicación final de las intervenciones, se adaptaron algunos instrumentos de evaluación y los materiales empleados, y se pilotearon ambas modalidades de atención, ya que una forma de aumentar la efectividad de las intervenciones consiste en adaptar los materiales de la intervención de acuerdo a las características de la población (Brown, Anderson, Schulte, Sintov \& Frissell, 2005).

Por otro lado, los análisis mostraron que tanto los adolescentes que recibieron el PIBA como los que recibieron el Consejo Breve, disminuyeron de manera significativa el promedio de tragos estándar consumidos y su nivel de riesgo, de la evaluación al seguimiento. También se observó una disminución significativa en el número de problemas relacionados y un incremento en el nivel de autoeficacia situacional. Además, ambas intervenciones alcanzaron un tamaño del efecto moderado, lo que coincidió con los estudios realizados en otros países (Winters et al., 2007).

Los resultados alcanzados para el grupo que recibió el PIBA, coinciden con los encontrados por Martínez, Salazar et al. (2008) con adolescentes de zonas urbanas en México, aunque, a diferencia de este estudio, se encontró un número menor de situaciones que alcanzaron diferencias estadísticas significativas en la autoeficacia situacional de los adolescentes. Algo importante de identificar es si existe alguna relación entre el tipo de situaciones de riesgo para el consumo y el número de caídas que se presentan durante la fase de seguimiento, aunque para ello se debe incrementar el número de participantes en ambas modalidades de intervención. Por otra parte, a diferencia de la investigación con estudiantes urbanos, en este trabajo se evaluó el nivel de riesgo del consumo para la ocurrencia de problemas agudos y no solo el promedio del consumo. 
Los resultados de los participantes que recibieron la sesión de Consejo Breve coincidieron con los datos reportados por Baer et al. (1992), quienes señalaron que el Consejo Breve y la intervención de varias sesiones, tenían un efecto significativo sobre la cantidad de tragos consumidos por los participantes durante el seguimiento. Lo que significa que el Consejo Breve puede ser tan efectivo como una intervención breve de mayor duración. Con todo, los individuos que participaron en el PIBA, tuvieron una mayor reducción en sus tasas de consumo, en comparación con la modalidad de una sola sesión.

\section{Conclusión}

Los resultados derivados de este estudio exploratorio inicial apuntan a que las intervenciones breves pueden ser efectivas para el trabajo con adolescentes estudiantes rurales usuarios de alcohol, en México. Se sugiere que ambas intervenciones se integren en un modelo escalonado de atención, donde el Consejo Breve sea la intervención mínima inicial, debido a su bajo costo y tiempo de aplicación, pero cuando esto no sea suficiente se ofrezca el PIBA (Sobell \& Sobell, 1999). De la misma forma, se puede dar oportunidad a los adolescentes de elegir la intervención. El modelo de la atención escalonada y las intervenciones breves plantean que cuando los individuos participan en la toma de decisiones relacionadas con el tratamiento, se incrementa la probabilidad de obtener resultados positivos (Miller \& Rollnick, 2002).

Sin embargo, a fin de incrementar la validez de ambas modalidades se sugiere ampliar el tamaño de la muestra, el período de seguimiento y comparar los resultados contra un grupo control. Algunas líneas de investigación pendientes son la adaptación de las intervenciones con adolescentes rurales no estudiantes y la identificación de qué intervención resulta más efectiva para cada grupo de adolescentes con base en su nivel de riesgo, ya que en este estudio solo se exploró, de manera inicial, la efectividad de ambas intervenciones, pero no permite asegurar cuál intervención es más efectiva para cada adolescente, de acuerdo a su consumo.

\section{Referencias}

Ayala, E., Cárdenas, G., Echeverría, L. \& Gutiérrez, M. (1998). Manual de autoayuda para personas con problemas en su forma de beber. México: Miguel Ángel Porrúa.

Baer, J., Marlatt, G., Kivlahan, D., Fromme, K., Larimer, M. \& Williams, E. (1992). An experimental test of three methods of alcohol risk reduction with young adults. Journal of Consulting and Clinical Psychology, 6, 974-979.

Berenzon, S., Medina-Mora, M. \& Lara, M. (2003). Servicios de salud mental: veinticinco años de investigación. Salud Mental, 26, 61-72.

Brown, S., Anderson, K., Schulte, M., Sintov, N. \& Frissell, K. (2005). Facilitating youth self-change through school-based intervention. Addictive Behaviors, 30, 1797-1810.

Calderón, G., Campillo, C. \& Suárez, C. (1981). Respuestas de la comunidad ante los problemas relacionados con el alcohol. México: Organización Mundial de la Salud/Instituto Mexicano de Psiquiatría.

Castro, M., Llanes, J., Margain, M. \& Carreño, A. (1999). Evaluación de planes de acción preventiva de riesgos psicosociales con el Modelo ChimalliDIF. Psicología Iberoamericana, 7, 5-13.

Clark-Carter, D. (2002). Investigación cuantitativa en Psicología: del diseño de experimentos al reporte de investigación. México: Oxford University Press.

Consejo Nacional contra las Adicciones. (2002). Modelos preventivos. México: CONADIC.

Kazdin, A. E. (2001). Métodos de investigación en Psicología Clínica. México: Pearson Educación.

Leukefeld, C., Godlaski, T., Hays, L. \& Clark, J. (1999). Developing a rural therapy with big city approaches. Substance Use EO Misuse, 34, 747-762.

Mariño, M., González-Forteza, G., Andrade, P. \& Medina-Mora, M. (1997). Validación de un cuestionario para detectar adolescentes con problemas por uso de drogas. Salud Mental, 1, 27-36.

Martínez, K., Pedroza, F. \& Salazar, M. (2008). Programa de intervención breve para adolescentes que 
abusan del consumo de alcohol y otras drogas: resultados de aplicación con adolescentes consumidores de alcohol de Aguascalientes y Distrito Federal. Anuario de Investigación en Adicciones, 9 , 46-57.

Martínez, K., Pedroza, F., Vacío, M., Jiménez, A. \& Salazar, M. (2008). Consejo Breve para adolescentes escolares que abusan del alcohol. Revista Mexicana de Análisis de la Conducta, 34, 247-264.

Martínez, K., Salazar, M., Pedroza, F., Ruiz, G. \& Ayala, H. (2008). Resultados prelimares del programa de intervención breve para adolescentes que inician el consumo de alcohol y otra drogas. Salud Mental, 31, 119-127.

Medina-Mora, M., Rascón, M., García, G. \& Ezbán, M. (1986). Patrones de consumo de alcohol y normas relacionadas con dicho consumo, en una población de Michoacán, México. Salud Mental, 9 (4), 87-91.

Medina-Mora, M., Rojas, E., Juárez, F., Berenzon, S., Carreño, S., Galván, J. et al. (1993). Consumo de sustancias con efectos psicotrópicos en la población estudiantil de enseñanza media y media superior de la República Mexicana. Salud Mental, 16 (3), 2-8.

Medina-Mora, M., Villatoro, J., López, E., Berenzon, S., Carreño, S. \& Juárez, F. (1995). Los factores que se relacionan con el inicio, el uso continuado y el abuso de sustancias psicoactivas en adolescentes mexicanos. Gaceta Médica de México, 131, 383-393.

Miller, W. R. \& Rollnick, S. (2002). Motivational interviewing: Preparing people to change addictive behavior (2a. ed.). New York: Guilford Press.

Natera, G. \& Tiburcio, M. (2007). Tailoring an intervention model to help indigenous families cope with excessive drinking in Central Mexico. Salud Mental, 30, 32-42.
Organización Panamericana de la Salud. Oficina Regional de la OMS. (2000). Guía internacional para vigilar el consumo del alcohol y sus consecuencias sanitarias. Washington, DC: Organización Mundial de la Salud.

Popham, R. (1976). Jellinek's international survey on drinking customs (Substudy No. 805). Canadá: Toronto Addiction Research Foundation.

Rodríguez- Martos, A. (2002). Efectividad de las técnicas de consejo breve. Adicciones, 14, 337-351.

Salazar, M. (2008). Evaluación de dos intervenciones breves para la disminución del consumo de alcohol en adolescentes rurales de Aguascalientes. Disertación doctoral no publicada, Universidad Nacional Autónoma de México, México DF.

Salazar, M., Álvarez, A. C., Galarza, C. \& Martínez, K. I. (2008). Inventario de situaciones de consumo de drogas en adolescentes rurales: construcción y características psicométricas. Revista de Psicología Social y Personalidad, 24 (2), 191-208.

Secretaría de Salud \& Instituto Nacional de Estadística, Geografía e Informática. (2002). Encuesta nacional de adicciones. Tabaco, alcohol y otras drogas. México: CONADIC.

Sobell, L., Maisto, S., Sobell, M. \& Cooper, A. (1979). Reliability of alcohol abusers self-reports of drinking behavior. Behavior Research and Therapy, 17, 157-160.

Sobell, M. \& Sobell, L. (1999). Stepped care for alcohol problems: An efficient method for planning and delivering clinical services. En J. Tucker \& G. Marlatt (Eds.), Changing addictive behavior: Bridging clinical and public health strategies (pp. 331-343). New York: Guilford Press.

Winters, C., Leitten, W., Wagner, E. \& O'Leary, T. (2007). Use of brief interventions for drug abusing teenagers within a middle and high school setting. The Journal of School Health, 17, 196-206. 
